\title{
Biomechanical \& Labor Protective Clothing Design
}

\author{
Shiwei $\mathrm{Li}^{1} \&$ Hong $\mathrm{Xie}^{2}$ \\ ${ }^{1}$ Shanghai University of Engineering Science, and Sports protection evaluation system of innovation project, China \\ Correspondence: Shiwei Li, Shanghai University of Engineering Science, and Sports protection evaluation system of \\ innovation project, China
}

Received: July 20, 2015

Accepted: August 12, 2015

Online Published: August 27, 2015

doi:10.5430/jbar.v4n2p20

URL: http://dx.doi.org/10.5430/jbar.v4n2p20

\begin{abstract}
The research on the labor protective clothing in China is in a backward stage, because there is no scientific theory and method to guide. Protective clothing in China is still in the stage of relying on experience. The study of labor protective clothing should take the human body as the center, and concentrate on the internal factors of body and external factors. Sports biomechanics can help researchers to further expand the research. The labor protective clothing combined with biomechanical has broken through conventional design of the labor protective clothing, which is able to reduce labor damage. The letter will show a new idea and principles for designing the labor protective clothing combined with biomechanical, which can provide guidance for the design of new labor protective clothing.
\end{abstract}

Keywords: Biomechanical, The labor protective clothing, Labor damage

\section{Introduction}

The design of labor protective clothing is to protect the workers from harm at work as much as possible, as well as to maximize in a measure of physiological, psychological, sign recognition, enterprise style reliability and work efficiency (Wu Weigang, 2001). Labor protective clothing design is to put safety in the first place, the labor protective clothing quality and the technical requirements are relatively high, as far as possible to eliminate the security threat of external environment on the human body.

At present, the conventional design of labor protective clothing is mainly based on experience, which is carried out the protective clothing functional design and some innovative design. The design of conventional labor protective clothing in addition to own conventional protection, but which does not put forward and promote the theories and methods. Such as Suli's coal miners protective clothing design, in which the labor protective clothing not only has the conventional protection function design, but also has the knees' and elbows' anti impact and lifesaving design.

\section{Study on the status of sports biomechanical}

\subsection{Study on the status of Chinese sports biomechanical}

Sang Chunlei(Sang Chunlei, 2013) studied on the driver bone collaterals muscles biomechanical modeling and seating comfort. The research contents included that establish cab and human muscle bone network models; study on parameters of influencing driver operational effectiveness of cab layout; study on the choice mechanism of driver lower extremity posture; the data was collected by equipment of the simulation experiment scientific analysis, whose analysis results show that the correlation is larger between each part of the joint torque and total muscle activity and cushion tangential force, and the principal component analysis method help to establish the comfort model.

Wu Xubo (Wu Xubo, 2014) studied on protective tennis clothing which is based on biomechanical .The research mainly on tennis course for upper extremity joints and muscles of protection. Systematic study disciplines is formed, which is about sports injuries, sports protection, motion capture, clothing materials, and so on. The system had been set up, which was about a relatively complete motion from the basic mechanics to development of protective product.

Wang Zhongzhen (Wang Zhongzhen, Ding Shuai, 2013) and others had studied and analyzed new impact-resistant protective clothing and fabrics on Chinese and foreign research, who had summed up the impact of protective effect of motion. Join material of absorbing shock and buffering capacity had been invented in the clothing or textiles. The protective clothing makes use of these special materials to absorb energy about collisions of objects, which can achieve the protective effect of the human body. 
Yang Ye (Yang Xiaoye, 2004) discussed about the possibility of using the full monitor of surface electromyography and analyses system for quadriceps trained, which could make a view to effectively determine the power of exercise training effect by relevant indicators, and to find athletes appear evaluation indexes of excessive training, and to judge the fatigue on this basis, but finally to prevent unnecessary sports injuries.

Guo Yanling (Guo Yanling, 2008) analyzed main contents, methods and techniques about the Chinese sports biomechanical. The object of Chinese sports biomechanical research is elite athletes, whose content is mechanical analysis on a specific movement of some technical movements. Research methods was adopted by combining experimental and theoretical models, which made a crossover study on mutual movement, such as biomechanical ,medicine and computers, and so on. Making use of motion capture and simulation is still the main means of technical analysis of movement.

\subsection{Study on the status of foreign sports biomechanical}

Foreign countries study on sports protection products had been quite mature, which is compared with the research in China. Adidas and Nike as the internationally renowned sports brands have carried out in-depth theoretical study about sports protective products, and formed a relatively mature technology to improve the performance of protective apparel.

US LP protective gear company developed a protective brace movement of muscle kinetic Safety Series, and created an ideal human pressure map which is based on different parts of the body and the principles of human exercise physiology and others. Make use of pressure in different parts of the body, and increase pressure graded in external body, which is able to form a new technology. Using this technique developed protective gear for the human joints, which can effectively reduce negative reaction time and energy losses caused by the body's internal factors (internal factors that can interfere with physical coordination), and increase the control of power during exercise, but also assist the muscles to keep in good condition and reduce unnecessary losses.

\section{Labor protective clothing design ideas}

Understand the status for the former research is necessary. Labor protective clothing design ideas researched on biomechanical was summed up. As the following labor protective clothing design ideas:

First, look for labor groups and conduct research, find the damage site of the body vulnerable muscles, joints or bones in labor process. Second, make careful analysis which is based on the human body sports injuries mechanism. According to biomechanical properties, the base model of labor protection is build. Third, seek to reflect the indexes of evaluation on biomechanical properties, such as strength, stiffness and hardness. Fourth, according to the indexes of evaluation on biomechanical properties, the right material whose combination is optimized is found, and the labor movement protection model is modified, which is beneficial to build human muscle or joint or bone network protection model. Fifth, protective gear made of different materials is conducted by the equipment of human dynamic measurement and analysis system of human movement, and the protection model is modified according to the concrete labor movement experimental data of human muscle or joint or bone network. Sixth, select the best material composition of protective effect on the human body, and design the initial labor protective clothing, and make protective performance test. Seventh, for other special protection design, such as the water saving airbag design, which need to make experiments and design labor protective clothing again, and so on. Eighth, make the overall protection performance evaluation for labor protective clothing with a protective effect, and select the best material composition of protective effect. Finally, evaluate the performance and optimize the design again.

As follows: 


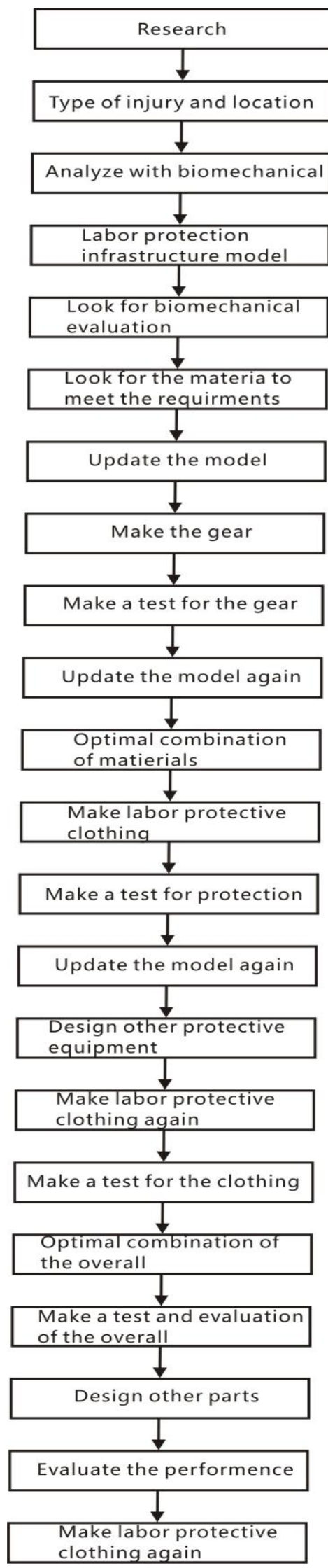




\section{The new design principle of the labor protective clothing}

National standards made general requirements which are "safe, practical, beautiful, generous" for protective clothing design. Meanwhile, the common principles of labor protective clothing design for work clothes design and ergonomics have formed in some industries. The main opinions summarized are the methods of work of the program, block foreign body, ergonomics, local improvement, corporate image and economy principle, and so on. As the following principles:

1. The simplified principle of structure. In the construction and coal industry, the labor protective clothing is different from everyday clothing which should reduce superfluous decoration, and avoid winding hanging, and reduce the maximum possibility of contacting with the environment objects as far as possible, such as stretch, twist, hanging with them. The living structures of labor protection clothing should be fully considered when it may be used. The labor protective clothing decoration mainly uses methods of color mosaic and heterogeneous mosaic and color segmentation and inlay line design, and so on. But when used, they has to be coordinated with the overall, and have the sense of the times, but which should avoid fashion.

2. Color management principles. Color design of ordinary labor protective clothing should be conducive to the health of workers and avoid affecting the operator's mood. According to the different characteristics of the occupations, the color theme and sentiment should be considered. In the dangerous working environment, high lightness and high saturation colors can be used in the labor protective clothing and hat, and so on. Color management can be applied to profession discrimination and job identification, which is able to play an important role in the comprehensive management of technology. Color design should not only coordinate with the work environment and color, but also beautify the company's image and corporate style.

The principles of biomechanical of labor protective clothing design is proposed which is based on the ordinary labor protection design principles, as follows:

1. Outline is dominated by H. Outline of $\mathrm{H}$ is succinct and capable. The outer contour of a rectangular shape has the strong sense of body. The application shows the characteristic of linear shape in the clothing, which have no waist, and the hip and chest is not prominent. The outer contour design is simple and sober and not cocky, whose characteristics are relaxed and casual and leisure. Outer contour should be designed according to actual needs, as well as other contour silhouette type, such as outline of $\mathrm{A}$, outline of $\mathrm{O}$ and outline of $\mathrm{X}$.

2. Coordinate with the local special design and overall. The new labor protective clothing can not avoid design the special protection in some parts, which is about to strengthen the protection of human body parts. Design for the special protection and the special composite fabric and increasing the special device, as well as of special structure, which may cause other structure designs. However, attentions should be paid to coordinate with the overall and the local special design. In addition, attentions should also be paid to stitch and sew the ordinary fabric with the special fabric, which may cause some problems.

3. Evaluation principles. Building evaluating indexes of labor protective clothing, which can make effectively detect and monitor in every aspect of the fashion design of labor protective clothing. In particular, the workers should inspect in the production process of special structure and reinforcement. The company should avoid the security threat for personnel, which need to prevent invalid or incomplete labor protection clothing. In addition, in order to make the labor protective clothing play its protective performance as much as possible, the enterprise should innovate the production process.

4. Value for money. Life safety is considered as the first protection criteria in the world. Reasonably select the labor protection fabric and carefully make labor protective clothing and avoid complex and difficult process. Safety first, then the money.

5. Safety protection design. Labor protective clothing design should follow natural green design concept of health and safety. From the different angle, the designer should consider security threats which come from external environment and body movement. The key value principle all throughout is man as the noumenon. In order to make the design meet the needs of the environment and safety, the design should be considered the labor protection clothing and environment as a whole.

\section{Summary}

This letter study the labor protective clothing which is on the basis of the sports protective clothing .The sports and labor protective clothing is based on the biomechanical .In order to make a tentative exploration for labor protection clothing design, the letter propose the relevant design ideas and design principles of labor protection clothing. 
The labor protective clothing design is a system engineering which is based on biomechanical. It is different from the routine of the former design. The new labor protective clothing design is paid attention to the theory innovation .The new theory can guides the design of the labor protective clothing. This research work is long and complex , and need more innovative practice.

\section{Acknowledgement}

The paper is founded by the Shanghai university of Engineering Science project. The project number is E1-0903-15-01081, and its title is 15KY0904.

\section{References}

Dong Shuting. (2012). Research on the design and development of non competitive bicycle sports apparel. Donghua University.

Guo Yanling. (2008). Research status and development trend of sports biomechanics in China. Sports Science Research, 2008 (12) :42-44.

Kuang Shicun, Guo Xia. Basic biomechanics about musculoskeletal system. Beijing: People's Medical Publishing House.

Lu Aiyun. (2010). Sports Biomechanics. Beijing: People's Education Press, 2010: 78-84.

Sang Chunlei. (2013). Biomechanical model of the bone of the driver and the study on the comfort of sitting posture. Jilin University China, 2013.

Su Li, Yu Wanqiao, Li Xiaoyin. (2010). Coal miners protective clothing. Modern Occupational Safety. 2010, 20 (12): 100-103.

Wang Zhongzhen, Ding Shuai. (2013). Research progress and design elements of impact resistant sport protective clothing. Shandong Textile Science \& technology, 2013 (1): 43-46.

Wu Weigang. (2001). Labor protective clothing and Ergonomic design. Journal of Tianjin University of Technology. 2001, 20 (3): 69-72.

Wu Xubo. (2014). Research on the protective tennis sports suit based on Biomechanics. Shanghai University of Engineering Science.

Yang Xiaoye. (2004). Fatigue evaluation of the surface EMG of the young men's quadriceps. Beijing Sport University.

Zhang Qian. (2014). Research on professional motorcycle sports protective clothing. Qilu University of Technology.

Zhang Ying. (2008). Design of motorcycle protective clothing. Soochow University.

Zhao Jin. (2009). Design and production of bicycle riding clothes. Soochow University. 